\title{
Weber-Fechner behavior in symmetry perception?
}

\author{
Peter A. van der Helm \\ Radboud University Nijmegen, Nijmegen, The Netherlands
}

\begin{abstract}
The literature contains several allusions to the idea that detection of (mirror) symmetry in the presence of noise follows the Weber-Fechner law. This law usually applies to first-order structures, such as length, weight, or pitch, and it holds that just-noticeable differences in a signal vary in proportion to the strength of the signal. Symmetry, however, is a higher order structure, and this theoretical note starts from the idea that, in noisy symmetry, the regularity-to-noise ratio defines the strength of the signal to be considered. We argue that the detectability of the symmetry follows a psychophysical law that also holds for Glass patterns. This law deviates from the Weber-Fechner law in that it implies that, in the middle range of noise proportions, the sensitivity to variations in the regularity-to-noise ratio is disproportionally higher than in both outer ranges.
\end{abstract}

\section{INTRODUCTION}

Within the general field of visual symmetry research (for reviews, see Tyler, 1996; van der Helm \& Leeuwenberg, 1996; Wagemans, 1997), this theoretical note investigates whether detection of (mirror) symmetry in the presence of noise follows the Weber-Fechner law (Fechner, 1860; Weber, 1834). This law holds that just-noticeable differences in a signal vary in proportion to the strength of the signal. Our claim (mine in collaboration with others in my lab) is that detection of noisy symmetry follows another psychophysical law that implies that, in the middle range of noise proportions, the sensitivity to variations in regularity-to-noise ratios is disproportionally higher than in both outer ranges. To avoid misunderstandings, keep in mind that the Weber-Fechner law is about change-tosignal ratios, not about regularity-to-noise ratios.

To elaborate our claim, we next introduce the topic of noisy-symmetry detection. Then, in Section 2, we discuss this psychophysical law and review the available empirical evidence on noisy-symmetry detection. Finally, in Section 3, by means of formal analyses, we compare this psychophysical law to the Weber-Fechner law.

\subsection{Imperfect Symmetry}

Symmetry is a visual regularity - that is, one of the regularities to which the visual systems of humans and many other species are sensitive (see, e.g., Barlow \& Reeves, 1979; Giurfa, Eichmann, \& Menzel, 1996; Horridge, 1996). Accordingly, symmetry detection is believed to be an integral part of the perceptual organization process that is applied to every incoming visual stimulus. In human perception research, for instance, symmetry has been shown to play a relevant role in issues such as object recognition (e.g., Pashler, 1990; Vetter \& Poggio, 1994), figure-ground segregation (e.g., Driver, Baylis, \& Rafal, 1992; Leeuwenberg \& Buffart, 1984; Machilsen, Pauwels, \& Wagemans, 2009), and amodal completion (e.g., Kanizsa, 1985; van Lier, van der Helm, \& Leeuwenberg, 1995).

The relevant point here is that natural symmetries are nearly always imperfect. In biology, for instance, the amount of asymmetry in the basically symmetrical shape of flowers and potential mates is believed to be a marker of genetic quality, and it is believed to influence pollinating and mating behaviors (see, e.g., Grammer \& Thornhill, 1994; Johnstone, 1994; Møller, 1992, 1995; Swaddle \& Cuthill, 1994; Thornhill \& Gangestad, 1994; Watson \& Thornhill, 1994). This indicates that it is both ecologically and perceptually relevant to assess how or how well the visual system deals with imperfect symmetry.

Also, repetition (e.g., van der Helm \& Treder, 2009) and Glass patterns (e.g., Dakin \& Bex, 2001; Earle, 1985; Glass, 1969; Glass \& Pérez, 1973; Glass \& Switkes, 1976; Khuu \& Hayes, 2005; Maloney, Mitchison, \& Barlow, 1987; Prazdny, 1984) are considered to be visual regularities, but here, we focus on imperfect symmetry. Sidelong, we do consider imperfect Glass patterns, which, as we discuss, behave similarly, but we do not consider imperfect repetition, which behaves differently (e.g., Bruce \& Morgan, 1975; Corballis \& Roldan, 1974; Csathó, van der Vloed, \& van der Helm, 2003; Fitts, Weinstein, Rappaport, Anderson, \& Leonard, 1956; Julesz, 1971; Treder \& van der Helm, 2007; van der Helm \& Leeuwenberg, 1996, 2004; van der Vloed, Csathó, \& van der Helm, 2005; Zimmer, 1984). Furthermore, at least three classes of imperfections can be distinguished.

First, a regularity slanted in depth yields systematic viewpoint-dependent imperfections in the proximal struc-

P. A. van der Helm, p.vanderhelm@donders.ru.nl 
ture of the regularity. Although this kind of imperfection is, of course, worth investigating, we do not consider it here, and we refer the reader to Sawada and Pizlo (2008), van der Vloed et al. (2005), and Wagemans, Van Gool, and d'Ydewalle (1991).

Second, a regularity may be imperfect because of relatively small displacements of corresponding points with respect to each other, which may arise due to jitter or morphing, for instance. Jitter and morphing are fine stimulus manipulations, but they also evoke an effect of the visual system's tolerance in matching potentially corresponding elements. Thereby, they are less suited to investigations of the Weber-Fechner issue, which requires an optimal control of the amounts of regularity and noise. This tolerance, by the way, is a general property of the visual system and not specific to symmetry perception, and we refer the reader to Barlow and Reeves (1979) and Dry (2008) for more details on how it affects symmetry perception (see also Rainville \& Kingdom, 2002).

Third, within the limits of the visual system's tolerance, a not necessarily spatially contiguous subset of the elements in a stimulus may form a perfect regularity to which the rest of the elements constitute noise (see Figure 1). This kind of imperfect regularity, which we consider here, is probably the one investigated most in research on regularity detection. By invoking the visual system's tolerance, it cannot only be generalized straightforwardly to stimuli in general but is also convenient for formal analyses because there is clarity about what, in such analyses, may be taken as regularity and noise. Notice that this clarity holds for the researcher who does the analyses; that is, it is not presumed to hold beforehand for the visual system, which does what it does for any incoming stimulus.

For the latter kind of imperfect regularity, it is clear that the regularity-to-noise ratio plays a decisive role, but less clear is its position in the psychophysical relationship between signal strength and the detectability of the regularity. Therefore, in Sections 2 and 3, we go into more detail on this psychophysical relationship and on the question of whether it complies with the Weber-Fechner law. To this end, however, we first have to assess what, in psychophysical analyses of regularity detection, is to be taken as the signal, the strength of which is needed in such analyses. This issue is addressed next.

\subsection{The Signal}

For a first-order structure such as length, weight, or pitch, it is clear what the signal is, and its detectability usually follows the Weber-Fechner law, except, perhaps, for extremely weak or strong signals. For regularities, however, the situation is different.

It may be tempting to take the remaining regularity in an imperfect symmetry or Glass pattern as the signal and to quantify its strength by the number of stimulus elements involved. However, symmetries and Glass patterns are higher order structures composed of structural relationships between the elements in a stimulus (see Figure 1). Symmetry arises due to the coherent relationships between elements forming symmetry pairs; that is, the virtual lines connecting elements in symmetry pairs are midpoint-colinear parallel lines (Jenkins, 1983). Likewise, Glass patterns arise due to the coherent orientations of dot dipoles (see also van der Helm \& Leeuwenberg, 1999; Wagemans et al., 1991; Wagemans, Van Gool, Swinnen, \& Van Horebeek, 1993). Hence, instead of quantifying the amount of regularity by the number of stimulus elements involved in a regularity, it is more appropriate to quantify it by the number of structural relationships that give rise to the regularity. This seems of little practical consequence, but it preludes the following.

In an imperfect symmetry, for instance, the amount of symmetry may have to cross a threshold for the symmetry to be perceptible, but even then, the percept is generally not simply "a perfect symmetry plus some perturbation." There is no physical "symmetry signal" emitted by the stimulus, and the symmetry percept can only result from the perceptual organization process which establishes the structural relationships that give rise to the symmetry. By all accounts, this perceptual organization process results in a percept (or neural code, if you will) in which the symmetry and the noise form one integrated whole. This integrated whole defines, in our view, the "signal" in formal analyses of regularity detection. This implies that one may still use the term symmetry signal, but only if it refers to the structural relationships that give rise to the symmetry
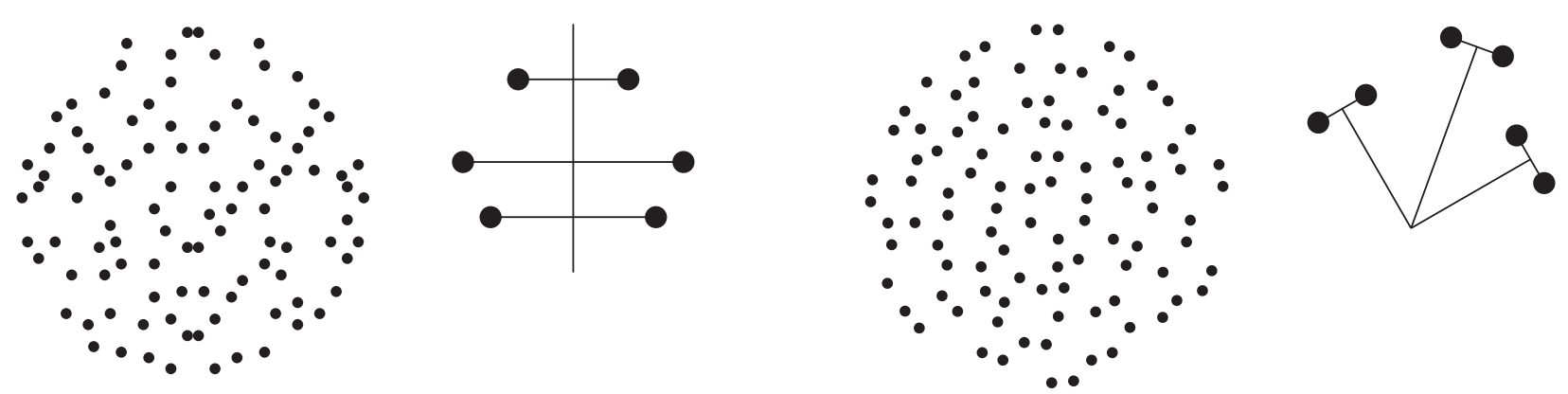

Figure 1. An imperfect symmetry with 40 symmetry pairs (at the left) and an imperfect Glass pattern with 40 dipoles (at the right). Despite the noise (20 unpaired dots), each regularity remains detectable due to the intact structural relationships that give rise to the regularity. In symmetry, these relationships are given by the midpoint-collinear and parallel virtual lines connecting elements in symmetry pairs, and, in Glass patterns, by the coherent orientation of the dot dipoles. 
with a strength modulated by the noise in this integrated whole. Our formal analyses need only this strength, by the way, and the foregoing implies that it is plausibly given by the ratio of the amount of regularity and the amount of noise in this integrated whole (see also Zanker, 1995).

Thus, in formal analyses of regularity detection, the regularity-to-noise ratio can be taken as the front end of the psychophysical relationship between signal strength and the detectability of the regularity. In the next section, we discuss what the position of the regularity-to-noise ratio seems to be in this psychophysical relationship.

\section{DETECTABILITY OF REGULARITY}

Various metrics of the detectability of symmetry in a stimulus have been proposed (see, e.g., Chipman, 1977; Dakin \& Watt, 1994; Dry, 2008; Masame, 1986, 1987; Yodogawa, 1982; Zimmer, 1984), and two things are pretty clear. First, a simple cross-correlation of the two symmetry halves does not seem to agree with human symmetry detection (see, e.g., Barlow \& Reeves, 1979; Tapiovaara, 1990). Second, most metrics capitalize on the concept of weight of evidence (MacKay, 1969); that is, they provide a measure of the weight of evidence for the presence of symmetry in a stimulus. This typically implies that, in such a metric, the somehow quantified amount of symmetry information in a stimulus is normalized by the somehow quantified total amount of information in the stimulus.

Here, to take a robust representative of such metrics, we focus on the one proposed in the so-called holographic approach to visual regularity (van der Helm \& Leeuwenberg, 1991, 1996, 1999, 2004). Like the traditionally assumed transformational approach advocated most prominently by Garner (1974) and Palmer (1983), the holographic approach is based on a mathematical characterization of visual regularity. Unlike the transformational formalization, however, the holographic formalization gives rise to a weight-of-evidence metric. Although most other metrics are tailored both to symmetry and to a specific stimulus type, they usually do not outperform this holographic metric, which is based on considerations of visual regularity in general and which, therefore, is more generally applicable (e.g., also to Glass patterns and repetition).

Without going into great detail, this holographic formalization implies that amounts of regularity are to be quantified as suggested above - that is, by the number of structural relationships that give rise to a regularity (see Figure 1). Accordingly, the holographic weight-ofevidence metric $(W)$ is defined by $W=E / n$, where $E$ is the amount of regularity in a stimulus and $n$ is the total number of elements in the stimulus.

Applied to symmetry, the holographic formalization implies that $E=R$, where $R$ is the number of structural relationships between elements forming symmetry pairs, or, in short, the number of symmetry pairs. Applied to Glass patterns, it implies that $E=R-1$, where $R$ is the number of dipoles, so that $R-1$ is the number of (nonredundant) structural relationships between the dipoles. Notice that $n=2 R$ for a perfect symmetry or Glass pattern. This does not hold for perfect repetition, in which case the holographic formalization implies that $\mathrm{E}$ is the number of repeats minus one, independent of the number of elements in each repeat. This implies that repetition is predicted to behave differently, which is in line with the empirical evidence cited in Section 1.1.

Furthermore, for an imperfect symmetry or Glass pattern, the total number of stimulus elements is $n=2 R+N$, where $N$ is the number of noise elements. This implies that the holographic weight of evidence for the presence of symmetry in an imperfect symmetry is given by $W=$ $R / n=R /(2 R+N)$. This formula can be rewritten into

$$
W=\frac{1}{2+N / R},
$$

which reveals the regularity-to-noise ratio $(R / N)$ as the decisive factor. Likewise, for an imperfect Glass pattern, $W=(R-1) /(2 R+N)$, which, for large $R$, also boils down to Equation 1.

The holographic weight of evidence $W$ for the presence of a regularity in a stimulus is proposed to be proportional to the detectability of the regularity. It is a function of $R / N$, which is in line with the idea that $R / N$ is to be taken as the signal strength (see Section 1.2). Furthermore, the detectability of a regularity (i.e., its discriminability from random patterns), or the strength of the regularity percept $(p)$, is typically operationalized by $d^{\prime}$ (Swets, 1964; Wickens, 2002). Hence, together, the proposed psychophysical relationship between signal strength and detectability is given by

$$
d^{\prime}=p=g W=g \frac{1}{2+N / R},
$$

where $g$ is the proportionality constant (which, by definition, is a multiplicative factor). As is usual in psychophysics, this $g$ can be seen as a parameter of the visual system, which also depends on stimulus type, so that it can only be determined experimentally (hence, its value cannot a priori be said to fall in a specific range). Its purpose in correlating predicted and empirical data points is to get more detailed fits than ordinal rankings.

In Section 3, we consider such fits in the formal comparison of Equation 2 with the Weber-Fechner law. Readers are free to jump directly to this comparison, but it is, of course, also relevant to assess the adequacy of the above analysis in human regularity detection. Therefore, in the following subsections, we first review the available empirical evidence on the detectability of symmetry and Glass patterns in the presence of noise.

\subsection{Imperfect Glass Patterns}

Maloney et al. (1987) studied the detectability of Glass patterns perturbed by randomly added noise dots. Participants had to discriminate random patterns from Glass patterns in which the number of dipoles $(R)$ varied from 10 to 200 and the number of randomly added noise dots $(N)$ varied from 25 to 2,000 . Using considerations from signal detection theory, they concluded that their data fit a model in which $d^{\prime}$ is proposed to be proportional to $1 /(2+N / R)$. Above, we concluded on a slightly different model; for large $R$, however, it boils down to Equation 2, which is identical to Maloney et al.'s model. 
Both models are equally based on the assumption that $d^{\prime}$ is proportional to the proposed term $1 /(2+N / R)$. Notice, however, that we developed this term on the basis of structural considerations about regularity in general (see van der Helm \& Leeuwenberg, 1996), whereas Maloney et al. (1987) developed it on the basis of signal detection considerations tailored to Glass patterns. Their considerations might be equally appropriate for imperfect symmetry, but not for imperfect repetition, which, as mentioned, behaves differently. In this respect, without challenging Maloney et al.'s approach, our approach can be said to put it in a broader perspective. Furthermore, precisely because Maloney et al. used different considerations, the two approaches provide converging theoretical evidence for the relevance of the term $1 /\left(2+N^{\prime} R\right)$ in case of Glass patterns.

Maloney et al. (1987) also noted that, by this term, the detectability of imperfect Glass patterns is predicted to remain constant when $R / N$ remains constant. After establishing the same for imperfect symmetry, we return to this point in Section 3, in which we also qualify Maloney et al.'s idea that it implies compliance with the WeberFechner law. Their data show further that Glass patterns degrade gracefully. In the next subsection, we begin to explicate this property for symmetry.

\subsection{Imperfect Symmetry}

2.2.1. Graceful degradation. Various empirical studies have shown that, perceptually, symmetry is quite robust to noise (see, e.g., Barlow \& Reeves, 1979; Masame, 1986, 1987; Troscianko, 1987; Wenderoth, 1995). The more specific question here, however, is not so much whether an imperfect symmetry still evokes a symmetry percept but, rather, how strongly noise affects the detectability of the remaining symmetry. This question may be addressed as follows.

Because $R=(n-N) / 2$, Equation 1 can be rewritten into $W=\left(1-N^{\prime} n\right) / 2$. This means that symmetry is pre-

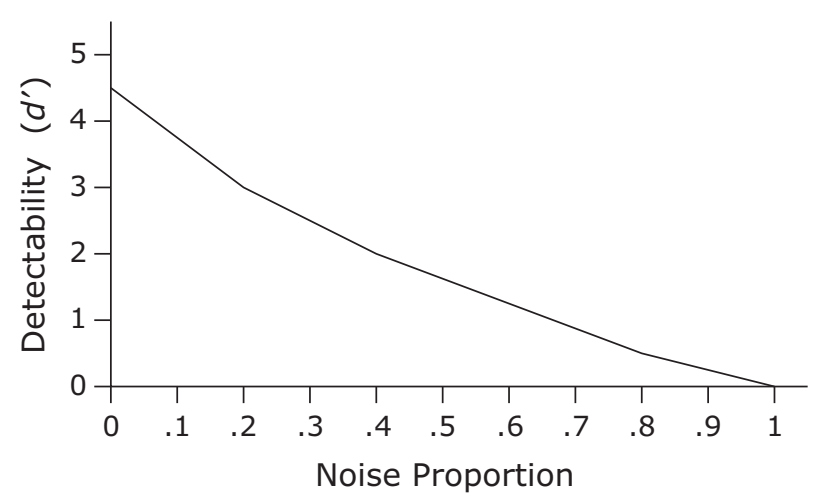

Figure 2. Summary of Barlow and Reeves's (1979) data for noisy symmetries on 100 dots. They had participants discriminate between different noise proportions, and from the resulting $d^{\prime} s$, they calculated the shown $d^{\prime}$ s for discrimination from random patterns; they also tested the latter separately, yielding consistent results. Detectability degrades gracefully as a function of the proportion of noise dots, with minor deviations from linearity for extreme noise proportions. dicted to degrade gracefully; that is, its detectability is predicted to get worse linearly with increasing noise proportion $N / n$. For instance, for constant $n$, one may replace an increasing number of symmetry pairs by pairs of noise elements. In that case, as is depicted in Figure 2, Barlow and Reeves's (1979) data confirm that symmetry does indeed degrade gracefully, just as Maloney et al.'s (1987) data show for Glass patterns.

Notice that this graceful degradation agrees with Olivers and van der Helm's (1998) finding that the detectability of a symmetrical target in a visual search display decreases with an increasing number of asymmetrical distractors. Notice further that, for extremely low and high noise proportions, Barlow and Reeves's data deviate slightly from linearity. As we discuss further in Section 3, Barlow and Reeves attributed these minor deviations to idiosyncracies in their stimulus material.

2.2.2. (A)symmetry effects. Several studies in the literature report that human observers seem to overestimate the amount of symmetry in an imperfect symmetry (see, e.g., Carmody, Nodine, \& Locher, 1977; Garner, 1970; King, Meyer, Tangney, \& Biederman, 1976). To our knowledge, the first systematic empirical study into this issue was conducted by Freyd and Tversky (1984). They concluded that, in cases of high levels of symmetry, the amount of symmetry indeed tends to be overestimated (which they called a symmetry effect), but that, in cases of low levels of symmetry, it tends to be underestimated (which they called an asymmetry effect). Using the same task and stimulus type but testing different conditions, Csathó, van der Vloed, and van der Helm (2004) came to another conclusion, however.

As indicated in Table 1 for the six conditions considered by Csathó et al. (2004), a pedestal imperfect symmetry with $W=W_{\mathrm{p}}$ can be made slightly more symmetrical by adding symmetry or removing noise, yielding $W=W_{\text {more }}$, and it can be made slightly less symmetrical by removing symmetry or adding noise, yielding $W=W_{\text {less }}$. Subsequently, by way of triadic comparisons, one may present participants with a pedestal imperfect symmetry plus a pair of less and more symmetrical variants (see Figure 3) and ask the participants which of the two variants is more similar to the pedestal. $W$ is proposed to be proportional to the strength of the symmetry percept, so that one predicts a symmetry effect if $W_{\mathrm{p}}$ is closer to $W_{\text {more }}$, and an asymmetry effect if $W_{\mathrm{p}}$ is closer to $W_{\text {less }}$.

In general, regarding these predictions, the overall level of symmetry is indeed relevant with a breaking point between low and high levels for $W_{\mathrm{p}} \approx .25$. More important, however, is that, at any level of symmetry, both symmetry effects and asymmetry effects are predicted to occur, depending on how $R / N$ is manipulated. This is shown in Table 1 for relatively high levels of symmetry, where Freyd and Tversky (1984) found only a symmetry effect. That is, a symmetry effect is predicted in Conditions 1, 3, and 5, but an asymmetry effect is predicted in Conditions 2, 4, and 6.

On the one hand, in Conditions 1 and 3, Csathó et al. (2004) found no (a)symmetry effect. Condition 1 was the 
Table 1

Triadic Comparisons Using a Pedestal Imperfect Symmetry With $W_{\mathrm{p}}=R / n$

\begin{tabular}{llll}
\hline & Less and More Symmetrical Variants & \multicolumn{1}{c}{ Weight of Evidence } & $\begin{array}{c}\text { Predicted } \\
\text { Effect }\end{array}$ \\
\hline 1 & Remove $d$ symmetry pairs & $W_{\text {less }}=(R-d) /(n-2 d)$ & symmetry \\
& Add $d$ symmetry pairs & $W_{\text {more }}=(R+d) /(n+2 d)$ & \\
2 & Add $d$ noise elements & $W_{\text {less }}=R /(n+d)$ & asymmetry \\
& Remove $d$ noise elements & $W_{\text {more }}=R /(n-d)$ & \\
3 & Replace $d$ symmetry pairs by noise elements & $W_{\text {less }}=(R-d) /(n-d)$ & symmetry \\
& Remove $d$ noise elements & $W_{\text {more }}=R /(n-d)$ & \\
4 & Add $d$ noise elements & $W_{\text {less }}=R /(n+d)$ & asymmetry \\
& Replace $d$ noise elements by symmetry pairs & $W_{\text {more }}=(R+d) /(n+d)$ & \\
5 & Add $d$ noise pairs & $W_{\text {less }}=R /(n+2 d)$ & symmetry \\
& Add $d$ symmetry pairs & $W_{\text {more }}=(R+d) /(n+2 d)$ & \\
6 & Remove $d$ symmetry pairs & $W_{\text {less }}=(R-d) /(n-2 d)$ & asymmetry \\
Remove $d$ noise pairs & $W_{\text {more }}=R /(n-2 d)$ & \\
\hline
\end{tabular}

Note-A symmetry effect is predicted if $W_{\mathrm{p}}$ is closer to $W_{\text {more }}$, and an asymmetry effect is predicted if $W_{\mathrm{p}}$ is closer to $W_{\text {less }}$. The predictions given here apply to $W_{\mathrm{p}}>.25$.

one with the smallest differences in $W$ between the pedestal and each of its variants, as well as between $W_{\text {more }}-W_{\mathrm{p}}$ and $W_{\mathrm{p}}-W_{\text {less }}$. This suggests that these differences may have been too small (i.e., below threshold) to evoke an (a)symmetry effect. Condition 3 was the one with the largest differences in $W$ between the pedestal and each of its variants, which suggests that the variants may have been nearly equally dissimilar to the pedestal — that is, too dissimilar to evoke an (a)symmetry effect.
On the other hand, Csathó et al. (2004) did find the predicted symmetry effect in Condition 5, as well as the predicted asymmetry effects in Conditions 2, 4, and 6. This suggests that, unlike Freyd and Tversky's (1984) suggestion, both symmetry and asymmetry effects may occur at the same level of symmetry. More important, it suggests that (a)symmetry effects are not the result of incorrect estimates of amounts of symmetry but, rather, the result of correct assessments of $R / N$.
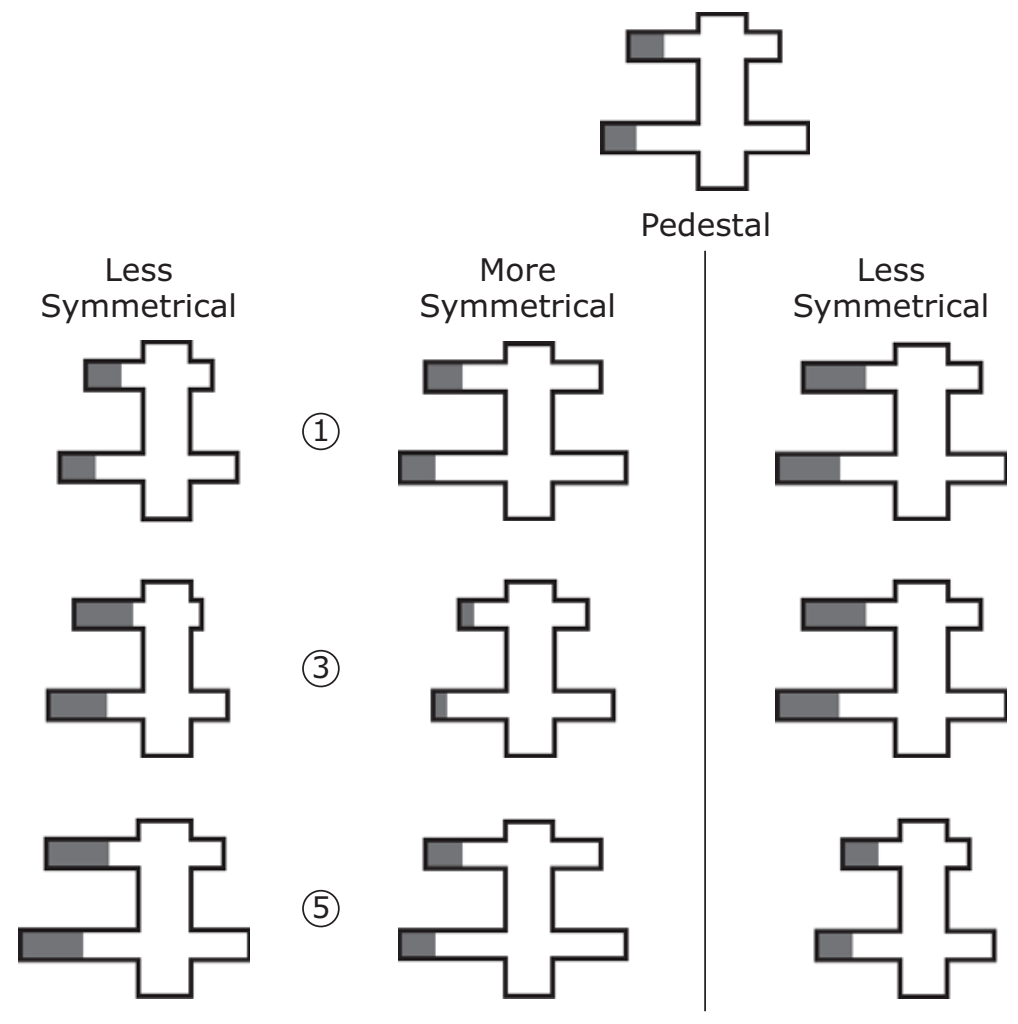

More

Symmetrical

(2)

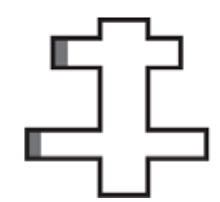

(4)

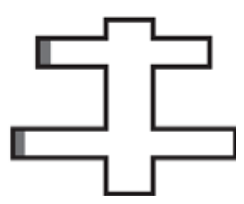

(6)

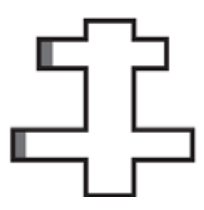

Figure 3. At the top, one of the five pedestal imperfect symmetries (with $W \approx .36$ ) in Csathó et al.'s (2004) triadic comparison experiment. Its limbs were shortened or lengthened to get pairs of less and more symmetrical variants. The shaded areas, which were white in the actual stimuli, indicate the noise. The six conditions are numbered as in Table 1, implying that a symmetry effect is predicted in Conditions 1,3, and 5, and that an asymmetry effect is predicted in Conditions 2, 4, and 6. 

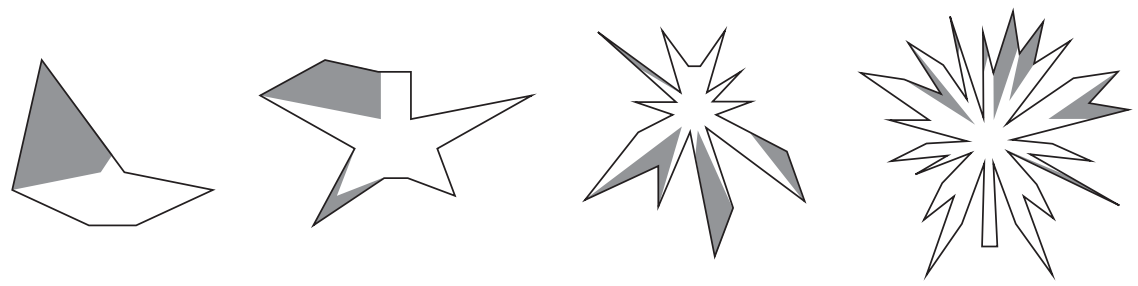

Figure 4. Sample of Olivers et al.'s (2004) imperfect symmetry stimuli-kindly provided by the authors-with $6,12,24$, and 48 contour segments, respectively. The white areas form the remaining symmetry, and the shaded areas form the noise. The actual stimuli were monochromatic.

2.2.3. No number effect. The detectability of perfect symmetry is known to be virtually independent of the number of stimulus elements (Baylis \& Driver, 1994; Csathó et al., 2003; Dakin \& Watt, 1994; Oomes, 1998; Tapiovaara, 1990; Wenderoth, 1996). This is in line with $W=E / n$ : In perfect symmetry, $n=2 E$, so that $W=$ $E / n=.5$, regardless of the number of stimulus elements. But what about imperfect symmetry? If not $n$ but $R / N$ is decisive, then also imperfect symmetry is predicted to show no number effect-at least as long as $R / N$ remains constant.

Olivers, Chater, and Watson (2004) challenged this prediction by testing imperfect symmetries, as given in Figure 4-that is, starlike shapes in which the number of contour segments $(n)$ could be $6,12,24$, or 48 . Starting from perfect symmetries, their perturbation manipulation implied that one sixth of the $n$ inward and outward pointing spikes were "pulled" inward or outward. They claimed that this yields $W=.33$ for all stimuli, and they asked participants to discriminate them from perfect symmetries. The error scores were the same for $n=6, n=12$, and $n=$ 24 but were higher for $n=48$, and they concluded that this goes against the holographic prediction.

However, as mentioned in Section 1.2, the percept of an imperfect symmetry is not an originally perfect symmetry plus some perturbation but, rather, results from the perceptual organization process applied to the entire stimulus. For instance, even knowing their manipulation, there is no way of telling for sure what the original perfect symmetries were in Figure 4. Hence, after perturbation, the stimuli have to be analyzed anew, which we did for the sample of 12 stimuli ( 3 for each value of $n$ ) we received from the authors.

That is, in van der Helm and Leeuwenberg (2004), we reanalyzed each stimulus by dividing the area inside the contour into a symmetry part and a noise part (see Figure 4). For each of the values $n=6, n=12$, and $n=$ 24 , our reanalysis resulted in $W \approx .30$, so that, here, we predict no number effect (i.e., no effect of $n$ ). For $n=48$, however, it resulted in $W \approx .40$, so that this case is predicted to be an outlier; this higher $W$ value arises, among other reasons, because some of the perturbed spikes were adjacent or symmetrically related, which reduces the perturbation effect per spike. Thus, we predict that discrimination of these stimuli from perfect symmetry is about equally good for $n=6, n=12$, and $n=24$, but worse for $n=48$. Hence, though Olivers et al. (2004) thought differently, their data were actually in fine agreement with the holographic prediction.

\subsection{Discussion}

The empirical data discussed in Sections 2.1 and 2.2.1 involved whole data curves (to our knowledge, the only ones in the literature). Furthermore, it is true that the data discussed in Sections 2.2.2 and 2.2.3 could be said to provide circumstantial evidence regarding the question at hand here, but these data do support the adequacy of the holographic metric $W$ for various stimulus types and in various experimental settings, including those, as in Section 2.2.2, in which participants were not asked explicitly for symmetry. Hence, taken together, the available empirical evidence provides converging evidence that Equation 1 is adequate for both imperfect symmetries and imperfect Glass patterns, and that Equation 2 is likely to represent the psychophysical relationship between signal strength and detectability. This suggests that $R / N$ is the decisive factor, in that it should remain constant to maintain a constant level of detectability. The latter may remind one of the WeberFechner law, with which regularity detection has indeed been associated. As we discuss next, however, this association needs qualification.

\section{WEBER-FECHNER BEHAVIOR?}

To address the necessary qualification of the association between regularity detection and the Weber-Fechner law, we start with the Weber-Fechner law itself, which is given by

$$
\Delta p=k \frac{\Delta S}{S},
$$

where $\Delta p$ is the change in the strength $p$ of a percept due to a change $\Delta S$ in the strength $S$ of a signal; the proportionality constant $k$ depends on stimulus type and is to be determined experimentally. Equation 3 means that changes $\Delta p$ in the strength of percepts remain constant when the change-to-signal ratio $\Delta S / S$ remains constant. In other words, for just-noticeable differences (JNDs) in a signal, it holds that $\Delta S / S$ is a constant (the so-called Weber fraction). That is, JNDs vary in proportion to the strength $S$ of the signal, so that a stronger signal has to change more for the change to be noticeable. This is known to hold for 
various first-order structures, such as weight, length, and pitch, if one excludes extremely weak and strong signals.

Integration of Equation 3, taken as differential equation $d p=k d S / S$, yields that strength $p$ of the percept is given by the nonlinear equation

$$
p=k \ln (S)+C,
$$

where $\ln$ is the natural logarithm and $C$ is another constant factor to be determined experimentally. It is true that, for nonextreme signal strengths, such a nonlinear relationship between percept $p$ and signal $S$ can also be approximated by Stevens's (1957) power law $p=q S^{a}$, where $q$ and $a$ are constants to be determined experimentally (the approximation may be achieved for $a<1$ ). However, Stevens's power law involves nothing more than data fitting, and it does not imply that changes in the strength of percepts remain constant when the changeto-signal ratio remains constant. The latter, in particular, is characteristic of Weber-Fechner behavior, as specified by Equations 3 and 4 .

In contrast to the Weber-Fechner law, which usually holds for first-order structures, $W=E / n$ was developed for higher order structures (i.e., for visual regularities). As we elaborated in Section 2, for imperfect symmetry and approximately for imperfect Glass patterns, $W=1 /(2+N / R)$, where $N$ is the number of noise elements, while $R$ is the number of symmetry pairs and dipoles, respectively. As expressed in Equation 2, $W$ is proposed to be proportional to the strength $p$ of the regularity percept, which, therefore, is predicted to be given by the nonlinear equation

$$
p=g W=g \frac{1}{2+N / R},
$$

where $g$ is the proportionality constant to be determined experimentally.

As is noted in Section 2.1, for imperfect Glass patterns, Maloney et al. (1987) found the same nonlinear relationship between $p$ and $R / N$. As we discuss next, they also alluded to Weber-Fechner behavior.

\subsection{Allusions to Weber-Fechner Behavior}

Maloney et al. (1987) first formulated the WeberFechner law as meaning that the detection threshold for some percept varies in direct proportion to the intensity of the background on which the percept appears. They then took regularity $R$ as the signal to be detected against noise $N$ as background. The latter does indeed yield a flavor of Weber-Fechner behavior, considering that Equation 5 implies that strength $p$ remains constant when $R / N$ remains constant. However, the Weber-Fechner law is about change-to-signal ratios (i.e., not about regularityto-noise ratios), and Maloney et al. did not assess compliance of their model with Weber-Fechner behavior, as specified by Equations 3 and 4. In van der Helm and Leeuwenberg (1996), we followed Maloney et al.'s allusion to Weber-Fechner behavior, but as we discuss below, we now argue differently.

Furthermore, for imperfect symmetry, Tjan and Liu (2005) proposed a so-called discrimination sensitivity metric $z$, and they too alluded to Weber-Fechner behavior.
That is, they used the Weber-Fechner Equation 4 (but then applied to lengths) to specify one of the components in $z$, and they argued that $z$ as a whole agrees with their empirical data. They therefore argued that, although their data "might seem to contradict" the Weber-Fechner law, they "were in fact consistent" with it (p. 897). Throughout their article, they stressed this consistency; but notice, first, that it refers to Weber-Fechner's law applied to lengths, and second, that their data and their metric $z$ as a whole clearly do not comply with Weber-Fechner's law applied to symmetry levels.

In fact, on the basis of two experiments, Tjan and Liu (2005) concluded that the visual system is disproportionally more sensitive to variations in lower levels of symmetry than to variations in higher ones (which goes against Weber-Fechner behavior). One might argue that their experimental method does not justify this as a conclusion specific to symmetry detection because, in these experiments, they used morphing to construct asymmetries. As we argued in Section 1.1, morphing is a fine manipulation, but it also introduces an effect of the visual system's tolerance in matching elements. This confounding factor, which is not specific to symmetry perception, might explain part of the variance in their data (see Barlow \& Reeves, 1979). However, as we discuss next, for the levels of symmetry they considered, the same seems to be implied by the holographic Equation 5, which finds the empirical support discussed in Section 2. This suggests that their conclusion is still tenable as a conclusion specific to symmetry detection.

\subsection{The Deviation From Weber-Fechner Behavior}

As we argued in Section 1.2, it seems appropriate to take not $R$ but $R / N$ as the strength $S$ of the signal that is to be considered in regularity detection. Now, substituting $S$ for $R / N$ in the holographic Equation 5 yields

$$
p=g \frac{1}{2+1 / S}
$$

and differentiation of this function $p$ of $S$ yields

$$
d p=g \frac{d S}{S} \frac{S}{(2 S+1)^{2}},
$$

where the multiplicative factor $S /(2 S+1)^{2}$ gives the deviation from the Weber-Fechner law. That is, this factor would have to be a constant to comply with Equation 3 .

As Figure 5 shows, however, this factor goes to zero for both extremes, and it peaks for $S=0.5$. Notice that $S=R / N$ is given in logarithmic scale, so that it displays the range from near-random patterns to near-perfect symmetries in a way that mirrors this range in terms of noise proportions (as is given in Figure 2). The relation between $S$ and noise proportion is not linear, but $S=$ 0.5 does correspond to a noise proportion of .5. Hence, the peak in this non-Weber-Fechner factor in terms of $S=R / N$ is in the middle range of noise proportions, for which Barlow and Reeves's (1979) data exhibited most strongly the predicted linear trend in terms of noise proportions (see Figure 2). 


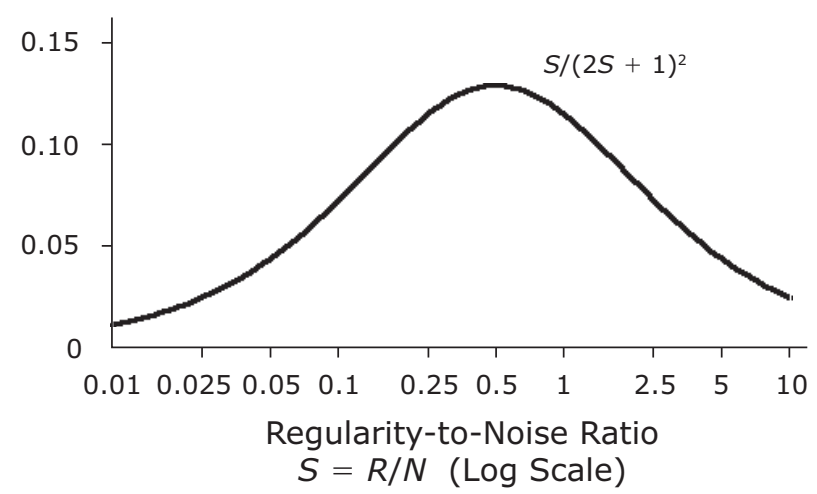

Figure 5. Graph of the multiplicative factor $S /(2 S+1)^{2}$, by which the holographic law in Equation 7 deviates from the WeberFechner law applied to the regularity-to-noise ratio $S=R / N$.

In other words, the non-Weber-Fechner factor in Figure 5 implies that, in the middle range of noise proportions, the sensitivity to variations in $R / N$ is disproportionally higher than in both outer ranges. That is, in this middle range, disproportionally smaller changes $\Delta S$ suffice to achieve the same change $\Delta p$. The disproportionally lower sensitivity in both outer ranges may also have triggered, in these outer ranges, more variance in Barlow and Reeves's (1979) data and may, thereby, have yielded the minor deviations from the predicted linearity in terms of noise proportions (see Figure 2).

Tjan and Liu (2005, p. 890) correctly assessed that the linearity in terms of noise proportions implies an equal discrimination of equal differences in noise proportion, but they contrasted it with their own conclusion, mentioned earlier, that discrimination is better for variations in lower levels of symmetry than for variations in higher ones. In one of their two morphing experiments, they considered variations at two levels of symmetry, and in the other experiment, they added a level in between. Due to properties inherent to morphing, variations in morphing cannot be translated one-to-one into variations in noise proportion, but the highest level was clearly near perfect symmetry and the lowest level was clearly in the middle range of noise proportions. Figure 5 shows that, in terms of $R / N$, our approach predicts a disproportionally higher sensitivity at the latter level, just as they argued their approach does; unlike our approach, their approach does not imply a decreasing sensitivity at still lower levels.

In another experiment, Tjan and Liu (2005) tested symmetrical dot patterns with randomly added noise dots. For this manipulation, they found that sensitivity to variations is actually best near perfect symmetry, and by means of a computational model, they explained this seemingly opposite result in terms of properties inherent to this manipulation (more stimulus variability for higher noise proportions). They argued that this finding agrees with Barlow and Reeves (1979), who (though more loosely) also attributed it to idiosyncracies in the stimulus material. However, in terms of Figure 2, this finding corresponds to the minor deviation from linearity for noise proportions smaller than .2; and notice that a same minor deviation occurs for noise proportions larger than .8. The latter deviation is not explained by Tjan and Liu's model which, as far as we can tell, does not explain the predominantly linear trend in Figure 2 either. In other words, in this experiment, Tjan and Liu can be said to have singled out an effect for near-perfect symmetry without qualifying it as one of the minor deviations at both extremities of the general trend.

We agree that idiosyncracies play a role, but the foregoing raises the question of whether they are responsible for opposite results, as Tjan and Liu (2005) claimed. After all, Figure 2 only shows minor deviations from the linear trend in terms of noise proportions, which we predicted starting from the same formula that predicts the disproportionally higher sensitivity to variations in $R / N$ in the middle range of noise proportions (see Figure 5). In other words, rather than speaking of opposite results, we would speak of results that may be slightly different due to idiosyncracies but still fit the general pattern predicted by this formula, without having to resort to allegedly decisive stimulus differences. To investigate this further, we did the following analysis.

\subsection{Fitting Barlow and Reeves's (1979) Data}

To further compare the Weber-Fechner Equation 4 and the holographic Equation 6, we fitted them both with $S=R / N$ to Barlow and Reeves's (1979) data. Because it is impossible to fit values approaching infinity (which, in these equations, arise for random patterns and perfect symmetries), we replaced their first and last data points with points obtained via linear interpolation between their first and last pairs of points, respectively. As is depicted in Figure 6, Equation 4 yields a best fit for $k=0.75$ and $C=2.34$, and Equation 6 yields a best fit for $g=7.64$, which is in the same order of magnitude as that Maloney et al. (1987) found for the same equation applied to Glass patterns. Both fits have a goodness of fit of $R_{a}^{2}=.96$, but notice that we were lenient to Equation 4: Choosing the interpolated first and last data points closer to the real ones would drop the fit for Equation 4 below $R_{a}^{2}=.70$ but would not affect the fit for Equation 6. Furthermore, as compared with Equation 4, Equation 6 is more parsimonious (i.e., it uses only one free parameter), and it also yields an $\mathrm{S}$ shape, which captures the qualitative trend in the data better.

Particularly for large $S$, the $\mathrm{S}$ shape predicted by Equation 6 deviates from the data. Just as Barlow and Reeves (1979) did, one could attribute this to stimulus idiosyncracies or, as we did in the previous subsection, to the disproportionally lower sensitivity in the outer ranges, but there is also a statistical angle. The fits above cover all 11 data points and suggest that, as compared with Equation 4, Equation 6 fits better for small $S$ but worse for large $S$. Indeed, if one fits only the first 6 data points (small $S$ ), Equations 4 and 6 yield a goodness of fit of $R_{a}^{2}=.90$ and $R_{a}^{2}=.99$, respectively; if one fits only the last 6 data points (large $S$ ), they yield $R_{a}^{2}=.98$ and $R_{a}^{2}=$ .89 , respectively. Hence, statistically, the equations are equivalent in the whole range from near-random patterns 

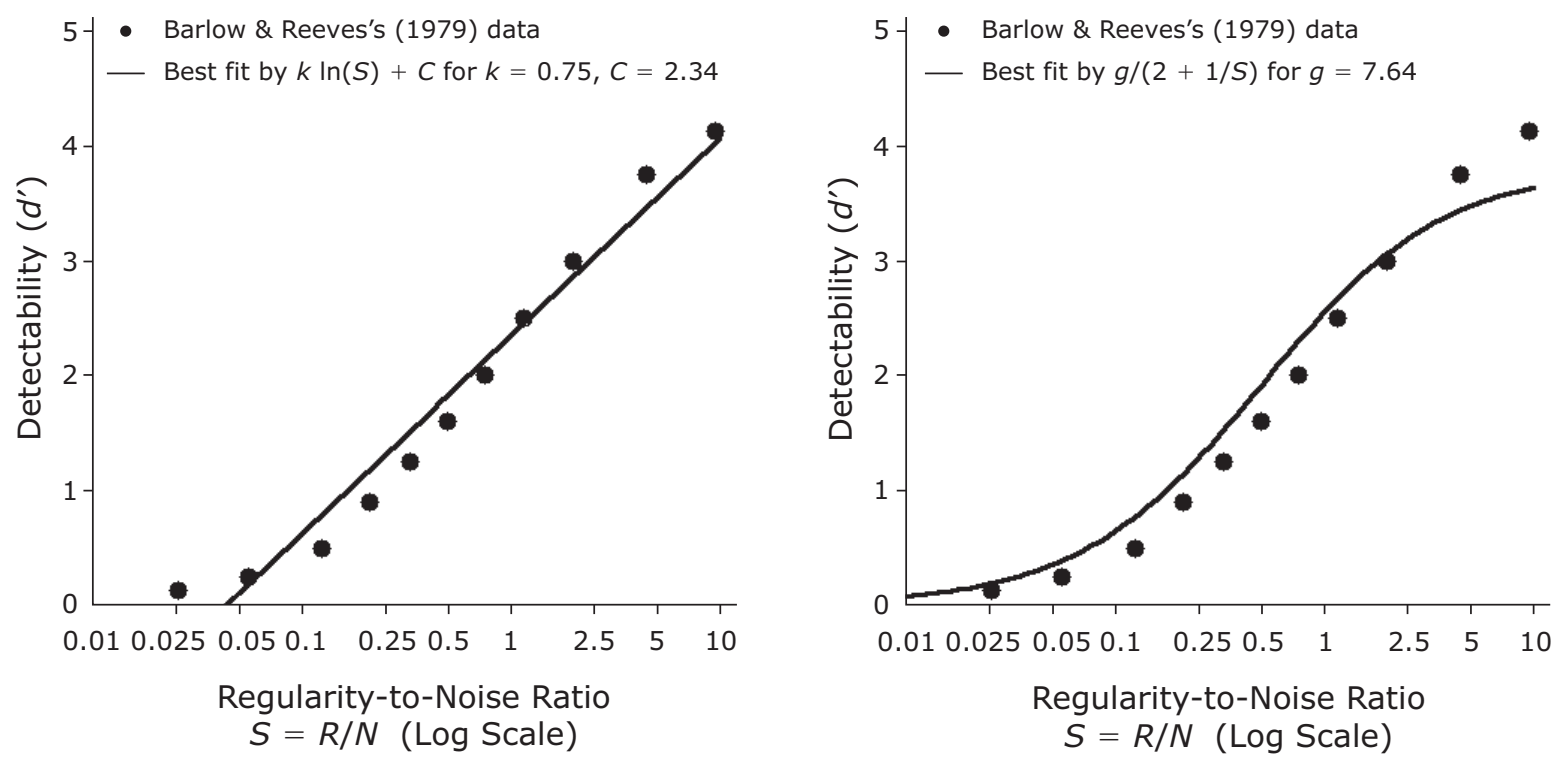

Figure 6. Fitting Barlow and Reeves's (1979) data on imperfect symmetry with $R$ symmetry pairs and $N$ noise elements, by the Weber-Fechner Equation 4 (at the left) and the holographic Equation 6 (at the right). Statistically, they yield the same goodness of fit $\left(R_{a}^{2}=.96\right)$, but the latter does so with only one instead of two parameters, and its $S$ shape reflects the trend in the data better.

to near-perfect symmetries, and they exhibit a trade-off between these limited ranges.

Given this statistical deadlock, the qualitative S-shaped correspondence between the data and Equation 6 is of the utmost importance. In contrast to Equation 4, Equation 6 captures the S-shaped trend in the data, not only in the middle range around $S=0.5$, but also in the two outer ranges, where it yields the obviously necessary floor and ceiling effects, which are not implied by the WeberFechner law; Tjan and Liu's (2005) approach implies a ceiling effect but no floor effect. This S-shaped correspondence also indicates that, unlike Tjan and Liu's suggestion, the different stimulus manipulations may yield slightly different, but not opposite, results due to idiosyncracies. That is, in our approach, their seemingly opposite results comply with one and the same formula without, as Tjan and Liu did, having to resort to allegedly decisive stimulus differences.

This analysis reveals a methodological issue, in that the free parameters $k$ and $C$ in Equation 4 can be chosen, such that Equation 4 approximates any of many equations with only one free parameter; hence, data may suggest WeberFechner behavior, even when the real behavior actually follows another rule. It also suggests that the holographic Equation 6 may well be this other rule - not only in cases of imperfect Glass patterns, as was also concluded by Maloney et al. (1987), but also in cases of imperfect symmetry.

\section{CONCLUSION}

For first-order structures, such as length, weight, or pitch, the Weber-Fechner law is known to fit poorly for extreme signal strengths, and, if one accepts this, one could maintain that it also holds for higher order structures, like symmetry. After all, as one might argue, the range over which a particular relation holds is almost always limited above and below. Also to our own surprise, however, our holographic law seems to do a better job, even though, excluding extreme signal strengths, the Weber-Fechner law yields the same goodness of fit. That is, our holographic law is not only more parsimonious but also accounts for the obvious floor and ceiling effects for extreme signal strengths.

Thereby, this theoretical note expands on Tjan and Liu (2005), who, for imperfect symmetry, advocated consistency with Weber-Fechner's law applied to lengths, but also provided evidence against this law applied to symmetry levels. We argue that our approach is more parsimonious than theirs, in that we captured their seemingly opposite results in one psychophysical law without resorting to allegedly decisive stimulus differences.

Furthermore, just as van der Helm and Leeuwenberg (1996) did, but this time within a more rigorous psychophysical setting, this theoretical note expands on Maloney et al. (1987) by showing that imperfect Glass patterns and imperfect symmetries follow the same psychophysical law. According to this law, the detectability of regularity $R$ in the presence of noise $N$ is proportional to $1 /(2+N / R)$. Unlike the Weber-Fechner law, this law implies that in the middle range of noise proportions, the sensitivity to variations in $R / N$ is disproportionally higher than in both outer ranges.

\section{AUTHOR NOTE}

I thank Matthias Treder for valuable comments on an earlier draft of this article. Correspondence concerning this article should be addressed to P. A. van der Helm, Donders Institute for Brain, Cognition, and Behaviour, Radboud University Nijmegen, Montessorilaan 3, 6525 HR Nijmegen, The Netherlands (e-mail: p.vanderhelm@donders.ru.nl). 


\section{REFERENCES}

Barlow, H. B., \& ReEves, B. C. (1979). The versatility and absolute efficiency of detecting mirror symmetry in random dot displays. Vision Research, 19, 783-793.

Baylis, G. C., \& Driver, J. (1994). Parallel computation of symmetry but not repetition within single visual shapes. Visual Cognition, 1, 377-400.

Bruce, V. G., \& Morgan, M. J. (1975). Violations of symmetry and repetition in visual patterns. Perception, 4, 239-249.

Carmody, D. P., Nodine, C. F., \& Locher, P. J. (1977). Global detection of symmetry. Perceptual \& Motor Skills, 45, 1267-1273.

Chipman, S. F. (1977). Complexity and structure in visual patterns. Journal of Experimental Psychology: General, 106, 269-301.

Corballis, M. C., \& Roldan, C. E. (1974). On the perception of symmetrical and repeated patterns. Perception \& Psychophysics, 16, 136142 .

Csathó, Á., van der Vloed, G., \& VAn der Helm, P. A. (2003). Blobs strengthen repetition but weaken symmetry. Vision Research, 43, 9931007.

Csathó, Á., van der Vloed, G., \& van der Helm, P. A. (2004). The force of symmetry revisited: Symmetry-to-noise ratios regulate (a)symmetry effects. Acta Psychologica, 117, 233-250.

DAKIN, S. C., \& BEX, P. J. (2001). Local and global visual grouping: Tuning for spatial frequency and contrast. Journal of Vision, 1, 99-111.

DAKIN, S. C., \& WATT, R. J. (1994). Detection of bilateral symmetry using spatial filters. Spatial Vision, 8, 393-413.

Driver, J., Baylis, G. C., \& Rafal, R. D. (1992). Preserved figureground segregation and symmetry perception in visual neglect. $\mathrm{Na}$ ture, 360, 73-75.

DRY, M. J. (2008). Using relational structure to detect symmetry: A Voronoi tessellation based model of symmetry perception. Acta Psychologica, 128, 75-90.

EARLE, D. C. (1985). Perception of Glass pattern structure with stereopsis. Perception, 14, 545-552.

Fechner, G. T. (1860). Elemente der Psychophysik [Elements of psychophysics]. Leipzig: Breitkopf \& Härtel.

Fitts, P. M., Weinstein, M., Rappaport, M., Anderson, N., \& LeONARD, J. A. (1956). Stimulus correlates of visual pattern recognition: A probability approach. Journal of Experimental Psychology, 51, 1-11.

FREYD, J., \& TVERSKY, B. (1984). Force of symmetry in form perception. American Journal of Psychology, 97, 109-126.

Garner, W. R. (1970). Good patterns have few alternatives. American Scientist, 58, 34-42.

GARNER, W. R. (1974). The processing of information and structure. Potomac, MD: Erlbaum.

Giurfa, M., Eichmann, B., \& Menzel, R. (1996). Symmetry perception in an insect. Nature, 382, 458-461.

GLASs, L. (1969). Moiré effect from random dots. Nature, 223, 578-580.

Glass, L., \& Pérez, R. (1973). Perception of random dot interference patterns. Nature, 246, 360-362.

Glass, L., \& SwitKes, E. (1976). Pattern recognition in humans: Correlations which cannot be perceived. Perception, 5, 67-72.

Grammer, K., \& Thornhill, R. (1994). Human (Homo sapiens) facial attractiveness and sexual selection: The role of symmetry and averageness. Journal of Comparative Psychology, 108, 233-242.

Horridge, G. A. (1996). The honeybee (Apis mellifera) detects bilateral symmetry and discriminates its axis. Journal of Insect Physiology, 42, 755-764.

JENKINS, B. (1983). Component processes in the perception of bilaterally symmetric dot textures. Perception \& Psychophysics, 34, 433-440.

Johnstone, R. A. (1994). Female preference for symmetrical males as a by-product of selection for mate recognition. Nature, 372, 172-175.

Julesz, B. (1971). Foundations of cyclopean perception. Chicago: University of Chicago Press.

Kanizsa, G. (1985). Seeing and thinking. Acta Psychologica, 59, 2333.

KhuU, S. K., \& Hayes, A. (2005). Glass-pattern detection is tuned for stereo-depth. Vision Research, 45, 2461-2469.

King, M., Meyer, G. E., Tangney, J., \& Biederman, I. (1976). Shape constancy and a perceptual bias towards symmetry. Perception \& Psychophysics, 19, 129-136.
LeEuWEnBerg, E., \& BufFart, H. (1984). The perception of foreground and background as derived from structural information theory. Acta Psychologica, 55, 249-272.

Machilsen, B., Pauwels, M., \& Wagemans, J. (2009). The role of vertical mirror symmetry in visual shape detection. Journal of Vision, 9(12, Art. 11), 1-11.

MACKAY, D. M. (1969). Information, mechanism and meaning. Cambridge, MA: MIT Press.

Maloney, R. K., Mitchison, G. J., \& Barlow, H. B. (1987). Limit to the detection of Glass patterns in the presence of noise. Journal of the Optical Society of America A, 4, 2336-2341.

Masame, K. (1986). Rating of symmetry as continuum. Tohoku Psychologica Folia, 45, 17-27.

Masame, K. (1987). Judgment of degree of symmetry in block patterns. Tohoku Psychologica Folia, 46, 43-50.

MøLler, A. P. (1992). Female swallow preference for symmetrical male sexual ornaments. Nature, 357, 238-240.

MøLlER, A. P. (1995). Bumblebee preference for symmetrical flowers. Proceedings of the National Academy of Sciences, 92, 2288-2292.

Olivers, C. N. L., Chater, N., \& Watson, D. G. (2004). Holography does not account for goodness: A critique of van der Helm and Leeuwenberg (1996). Psychological Review, 111, 242-260.

Olivers, C. N. L., \& VAN DER Helm, P. A. (1998). Symmetry and selective attention: A dissociation between effortless perception and serial search. Perception \& Psychophysics, 60, 1101-1116.

Oomes, S. (1998). Human visual perception of spatial structure: Symmetry, orientation, and attitude. Unpublished doctoral dissertation, Radboud University Nijmegen, Nijmegen, The Netherlands.

PALmer, S. E. (1983). The psychology of perceptual organization: A transformational approach. In J. Beck, B. Hope, \& A. Rosenfeld (Eds.), Human and machine vision (pp. 269-339). Orlando, FL: Academic Press.

PASHler, H. (1990). Coordinate frame for symmetry detection and object recognition. Journal of Experimental Psychology: Human Perception \& Performance, 16, 150-163.

Prazdny, K. (1984). On the perception of Glass patterns. Perception, 13, 469-478

Rainville, S. J. M., \& Kingdom, F. A. A. (2002). Scale invariance is driven by stimulus density. Vision Research, 42, 351-367.

SAWADA, T., \& Pizlo, Z. (2008). Detection of skewed symmetry. Journal of Vision, 8(5, Art. 14), 1-18.

Stevens, S. S. (1957). On the psychophysical law. Psychological Review, 64, 153-181.

Swaddle, J. P., \& Cuthill, I. C. (1994). Preference for symmetric males by female zebra finches. Nature, 367, 165-166.

Swets, J. A. (ED.) (1964). Signal detection and recognition by human observers: Contemporary readings. New York: Wiley.

TAPIOVAARA, M. (1990). Ideal observer and absolute efficiency of detecting mirror symmetry in random images. Journal of the Optical Society of America A, 7, 2245-2253.

Thornhill, R., \& GANGESTAD, S. W. (1994). Human fluctuating asymmetry and sexual behavior. Psychological Science, 5, 297-302.

TJAN, B. S., \& LIU, Z. (2005). Symmetry impedes symmetry discrimination. Journal of Vision, 5, 888-900.

Treder, M. S., \& VAN DER Helm, P. A. (2007). Symmetry versus repetition in cyclopean vision: A microgenetic analysis. Vision Research, 47, 2956-2967.

Troscianko, T. (1987). Perception of random-dot symmetry and apparent movement at and near isoluminance. Vision Research, 27, 547554.

TyleR, C. W. (1996). Human symmetry perception. In C. W. Tyler (Ed.), Human symmetry perception and its computational analysis (pp. 3-22). Mahwah, NJ: Erlbaum.

VAN DER Helm, P. A., \& LeEUWENBERG, E. L. J. (1991). Accessibility: A criterion for regularity and hierarchy in visual pattern codes. Journal of Mathematical Psychology, 35, 151-213.

van der Helm, P. A., \& Leeuwenberg, E. L. J. (1996). Goodness of visual regularities: A nontransformational approach. Psychological Review, 103, 429-456.

van der Helm, P. A., \& Leeuwenberg, E. L. J. (1999). A better approach to goodness: Reply to Wagemans (1999). Psychological Review, 106, 622-630. 
van der Helm, P. A., \& Leeuwenberg, E. L. J. (2004). Holographic goodness is not that bad: Reply to Olivers, Chater, and Watson (2004). Psychological Review, 111, 261-273.

van der Helm, P. A., \& Treder, M. S. (2009). Detection of (anti)symmetry and (anti)repetition: Perceptual mechanisms versus cognitive strategies. Vision Research, 49, 2754-2763.

van der Vloed, G., Csathó, Á., \& VAn der Helm, P. A. (2005). Symmetry and repetition in perspective. Acta Psychologica, 120, 74-92.

van Lier, R. J., van der Helm, P. A., \& LeEuwenberg, E. L. J. (1995).

Competing global and local completions in visual occlusion. Journal of Experimental Psychology: Human Perception \& Performance, 21, 571-583.

Vetter, T., \& Poggio, T. (1994). Symmetric 3D objects are an easy case for 2D object recognition. Spatial Vision, 8, 443-453.

WaGemans, J. (1997). Characteristics and models of human symmetry detection. Trends in Cognitive Sciences, 1, 346-352.

Wagemans, J., Van Gool, L., \& D'Ydewalle, G. (1991). Detection of symmetry in tachistoscopically presented dot patterns: Effects of multiple axes and skewing. Perception \& Psychophysics, 50, 413-427.

Wagemans, J., Van Gool, L., Swinnen, V., \& Van HorebeeK, J. (1993). Higher-order structure in regularity detection. Vision Research, 33, 1067-1088.
Watson, P. J., \& Thornhill, R. (1994). Fluctuating asymmetry and sexual selection. Trends in Ecology \& Evolution, 9, 21-25.

WeBER, E. H. (1834). De subtilitate tactus [On the sensitivity of the touch sense]. In E. H. Weber, De pulsu, resorptione, auditu et tactu. Annotationes anatomicae et physiologicae (pp. 44-174). Leipzig: Koehler.

WENDEROTH, P. (1995). The role of pattern outline in bilateral symmetry detection with briefly flashed dot patterns. Spatial Vision, 9, 57-77.

Wenderoth, P. (1996). The effects of dot pattern parameters and constraints on the relative salience of vertical bilateral symmetry. Vision Research, 36, 2311-2320.

WiCKEns, T. D. (2002). Elementary signal detection theory. New York: Oxford University Press.

YodogawA, E. (1982). Symmetropy, an entropy-like measure of visual symmetry. Perception \& Psychophysics, 32, 230-240.

ZANKER, J. M. (1995). Does motion perception follow Weber's law? Perception, 24, 363-372.

Zimmer, A. C. (1984). Foundations for the measurement of phenomenal symmetry. Gestalt Theory, 6, 118-157.

(Manuscript received January 8, 2010;

revision accepted for publication April 26, 2010.) 